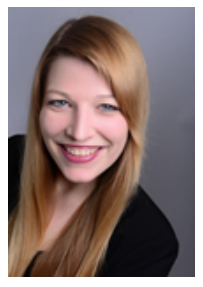

Charlotte Förster

Juniorprofessorin

für Europäisches Management, Technische Universität Chemnitz

charlotte.foerster@wiwi.tu-chemnitz.de

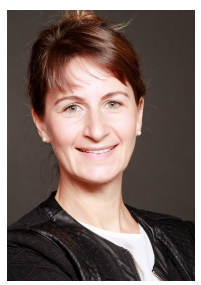

Nina Füreder

Wissenschaftliche Mitarbeiterin am Institut für Leadership \& Change Management, JKU Linz

nina.fuereder@jku.at

\title{
Wie können Führungskräfte zur Resilienz von (Gesundheits)Organisationen beitragen?
}

\author{
Lehren aus der aktuellen COVID-19 Krise
}

Der Ausbruch von SARS-CoV-2 stellt für unser Gesundheitssystem eine Feuerprobe dar, die weltweit bisher nur wenige bestanden haben. Trotz eingehender Warnungen durch die WHO vor einer globalen Grippe Pandemie, Ebola oder einer nicht näher spezifizierten unbekannten Krankheit ${ }^{1}$ sowie dem Risiko eines bioterroristischen Anschlags, scheint kaum ein Gesundheitssystem in Europa auf eine Pandemie vorbereitet zu sein. Eine konsequente Unterschätzung von COVID-19, ein Flickenteppich an Maßnahmen der Politik sowie fehlende Koordination und Konsequenz haben einerseits zu hohen Infektionszahlen beigetragen, anderseits auch zu einem Zustand des Dauer-(Nicht)-Lockdowns, gesellschaftlichen Missmuts, einer Gefährdung der öffentlichen Gesundheit sowie einer wirtschaftlich-finanziellen Katastrophe. In Zeiten wie diesen ist die Resilienz von Organisationen des Gesundheitssystems, definiert als die Fähigkeit mit kritischen Situationen und Krisen effektiv umgehen zu können und daran im besten Fall zu wachsen, besonders wichtig. Beachtet man die Rolle der Führungskraft in der organisationalen Krise sowie den Einfluss der Führungskraft auf die Gesundheit, das Wohlbefinden und die Produktivität ihrer MitarbeiterInnen, so stellt sich die Frage welchen Beitrag die Führungskraft zur Resilienz von (Gesundheits)Organisationen leisten kann. In einer Studie wurden vier Handlungsfelder identifiziert. Diese sind (1) Antizipation und ein offenes Mindset, (2) Strukturelle Probleme, (3) Vernetzung sowie (4) Individuelle Resilienzstrategien, welche Führungskräfte stärker berücksichtigen sollten, um zukünftig besser auf Krisen vorbereitet zu sein. 


\section{Resiliente Organisationen und die Rolle der Führungskraft}

Dramatische Waldbrände in Australien, die Explosion im Hafen von Beirut, die Ermordung von George Floyd, COVID-19; das Jahr 2020 lässt sich als das Jahr der Krisen beschreiben. ${ }^{2}$ Obwohl nur die wenigsten dieser Krisen per se Unternehmenskrisen waren, sind Organisationen häufig stark davon betroffen. Um in einer solch krisenanfälligen

\section{Organisationale Resilienz}

Organisationale Resilienz beschreibt die Fähigkeit Trends und Bedrohungen zu antizipieren, unerwartete Ereignisse effektiv zu bewältigen und aus diesen Ereignissen zu lernen. ${ }^{3}$ Duchek (2020) unterscheidet drei Phasen der organisationalen Resilienz: 1) Antizipation, 2) Bewältigung sowie 3) Anpassung. Während sich die erste Phase, Antizipation, vor dem Eintreten der eigentlichen Krise ereignet und primär die Fähigkeit eines Unternehmens umfasst, die Umgebung zu scannen, kritische Entwicklungen zu identifizieren und sich auf potenzielle Krisen vorzubereiten, betrifft die zweite Phase, die Bewältigung, die tatsächliche Krisensituation. In dieser Phase geht es einerseits darum die Krise zu akzeptieren (nicht jede Krise lässt sich verhindern) sowie andererseits Lösungen für bestimmte Probleme zu entwickeln und diese Lösungen rechtzeitig umzusetzen. Die letzte Phase, die Anpassung, bezieht sich auf die Zeit nach der Krise. Hier ist es wichtig, dass die Organisation die Krise reflektiert, hieraus lernt und diese Erkenntnisse in entsprechenden Veränderungen umsetzt, um auf diese Weise besser auf zukünftige Krisen vorbereitet zu sein.

Welt zu überleben, in der hinter jeder Ecke eine potenzielle Krise lauert, müssen Unternehmen daher äußerst resilient sein.

In der Forschung wird organisationale Resilienz häufig als Ergebnis verstanden, wohingegen neuere Studien Resilienz eher als dynamischen Prozess definieren. ${ }^{4}$ Darüber hinaus weist die aktuelle Resilienzforschung daraufhin, dass es sich bei der Resilienz um ein mehrstufiges Konstrukt handelt. ${ }^{5}$ Um die organisationale Resilienz von einer ganzheitlichen Perspektive zu betrachten, muss somit auch die individuelle Ebene sowie die Team- und Führungsebene betrachtet werden, wobei natürlich besonders die individuellen Ressourcen für die organisationale Resilienz wichtig sind. ${ }^{6}$ Beachtet man die Rolle der Führungskraft in der organisationalen Krise ${ }^{7}$ sowie den Einfluss der Führungskraft auf die Gesundheit, das Wohlbefinden und die Produktivität ihrer Mitarbeiterlnnen ${ }^{8}$, so stellt sich die Frage welchen Beitrag die Führungskraft zur Resilienz von Organisationen leisten kann. Wirft man einen Blick in die aktuelle Resilienz-Literatur, so zeigt sich, dass die Beziehung zwischen der Führungskraft und der Resilienz von Organisationen bisher eher vage beschrieben ist. ${ }^{9}$

\section{Das Krankenhaus im System der Gesundheitsversorgung}

Die Entwicklung von einer Hilfseinrichtung und Pilgerherberge zu einer hochtechnologisierten und spezialisierten Einrichtung, beschreibt den wohl komplexesten Organisationstyp unserer Zeit. ${ }^{10}$ Das Krankenhaus lässt sich dahingehend als mehrdeutige, unkontrollierbare Organisation beschreiben, in welchem verschiedene „Zielkomplexe“ und kulturelle Orientierungen auf konfliktträchtige Weise aufeinandertreffen. Die Medizin 
(ÄrztInnen) ist beispielsweise auf die professionell abgestimmte, rationale Gestaltung des Behandlungsgeschehens fokussiert. Die pflegerische Versorgung zielt darauf ab die Bedürfnisse der PatientInnen zu befriedigen, sie zu schützen, aber dennoch auch zu überwachen. Beim Zielkomplex Lehre, Aus- und Weiterbildung stehen die Mitglieder selbst im Zentrum der Aktivitäten, wohingegen die Forschung die Produktion sachbezogener Erkenntnisse ansteuert. Die administrativ-betriebliche Selbsterhaltung (Controlling, Qualitätsmanagement) beinhaltet hingegen organisationsbezogene Handlungsstrategien, die sich allerdings als äußerst bürokratisch und reglementierend beschreiben lassen und daher oft wie ein „Fremdkörper" gesehen werden. In der Organisation Krankenhaus treffen somit verschiedenste Zielkonflikte aufeinander, die noch dazu in unterschiedlichen und darüber hinaus recht unabhängigen Hierarchien organsiert sind. In diesem Sinne besteht die Krankenhausleitung in der Regel aus der ärztlichen Direktion, der Pflegedirektion und der Verwaltungsdirektion, welche tendenziell unterschiedliche Interessen vertreten. Besonders ist, dass diese Hierarchieebenen sich teilweise überlappen oder sogar untereinander in Konkurrenz stehen, wenn es beispielsweise um die Verteilung von Ressourcen geht. Die Herausforderung besteht somit in der permanenten Bewältigung dieser fundamentalen Zielkonflikte sowie der Verbindung der sich dadurch herausbildenden organisationalen Subkulturen. Neben diesen Zielkonflikten treten zudem kritische Situationen in Krankhäusern oft unvorhersehbar auf, dies erfordert, dass die Organisationen mit ihren Führungskräften und den dazugehörigen Teams die Fähigkeit besitzen Probleme zu antizipieren, Verfahren flexibel anzupassen und konkurrierende Anforderungen priorisieren zu können, um ein Grundniveau der Routineversorgung in jeglicher Lebenslage aufrechterhalten zu können.

\section{Herausforderungen und die Rolle der Resilienz von Gesundheitsorganisationen}

Gesundheitsorganisationen im Allgemeinen und Krankenhäuser im Besonderen stehen vor vielfältigen Herausforderungen, wenn es darum geht, erschwingliche Pflege, optimierte Behandlungsprozesse und Innovationen anzubieten, um auch angesichts der sich verändernden Umgebung und Rahmenbedingungen die Qualität und Sicherheit der Gesundheitsversorgung zu gewährleisten. Trotz vieler Bemühungen haben sich über die Jahre einige tiefgreifende Strukturprobleme herausgebildet, wie beispielsweise der chronische Personalmangel in der Pflege, sowie die fehlende Digitalisierung und Vernetzung. Diese strukturellen Probleme wurden im vergangenen Jahr durch die auftretende COVID-19 Pandemie noch zusätzlich erschüttert. So galt es, trotz begrenztem medizinischen Wissen COVID-19 Erkrankte zu behandeln, trotz mangelnder Testmöglichkeiten Diagnosen zu stellen und trotz fehlendem Schutzmaterial Personal und PatientInnen zu schützen. Gleichzeitig mussten quasi über Nacht zusätzliche Betreuungseinheiten für Infizierte geschaffen werden, ohne andere Patienten zu benachteiligen. Handlungsanweisungen und Prozesse waren an die Dynamik des Pandemieverlaufs und die rasche Wissensvermehrung anzupassen. 
Auch wenn Krisen, welche die Organisationen im Gesundheitssystem in der Vergangenheit betrafen, tendenziell kurzfristiger Natur waren, denkt man zum Beispiel an das Seilbahnunglück von Kaprun zurück, so hat die aktuelle COVID-19 Pandemie gezeigt, dass Organisationen auch länger andauernde Krisen überstehen müssen. Dies bedeutet, dass Krankenhäuser und ihre Mitglieder nicht nur kurzfristig, sondern auch langfristig resilient sein müssen, um Krisen jedweder Art nicht nur effizient, sondern insbesondere auch gesund zu überstehen. Betrachtet man Resilienz als einen sowohl proaktiven als auch reaktiven Ansatz, so gilt es eine Krise bzw. mögliche Gefahr frühzeitig zu erkennen und diese im besten Fall sogar zu verhindern, anderseits aber auch in der Krise flexibel zu reagieren und aus dieser zu lernen. Berücksichtigt man weiterhin die unterschiedlichen sozialen, kulturellen und organisatorischen Faktoren, welche die Arbeit im Gesundheitssystem prägen, so lässt sich die Resilienz von Gesundheitsorganisationen als ein mehrstufiges Konstrukt beschreiben, bei dem die Resilienz der verschiedenen Organisationsebenen stark miteinander verbunden sind, wobei den Führungskräften eine Schlüsselfunktion zugeschrieben werden kann.

\section{Die Rolle der Führungskraft für die Resilienz in Gesundheitsorganisationen}

Führungskräfte im Gesundheitswesen, als Hauptverantwortliche im organisatorischen Handeln, tragen die Verantwortung für die Festlegung und Umsetzung kurz- und langfristiger Ziele. Mit Verständnis und Gefühl für alle im Krankenhaus relevanten Themen, schaffen sie den Spagat zwischen Wirtschaftlichkeit und Patientenwohl.

Obwohl die Rolle individueller Ressourcen für die organisationale Resilienz ${ }^{11}$ als auch der Einfluss von Führungskräften auf die MitarbeiterInnen ${ }^{12}$ bekannt ist, erscheint die Rolle der Führungskraft in der organisationalen Resilienzforschung als tendenziell vernachlässigt. ${ }^{13}$ Da Führungskräfte nicht nur Strukturen und Prozesse gestalten, sondern auch ein Umfeld schaffen, in dem Individuen und Teams resilientes Verhalten entwickeln ${ }^{14}$, ist es wichtig zu untersuchen, wie Führungskräfte im Gesundheitswesen ihre Teams und Organisationen erfolgreich durch die COVID-19 Krise navigieren können. Dar-

\section{Studie Organisationale Resilienz}

Da Organisationen im Gesundheitssystem insbesondere in der Krise stark durch ihre Führungskräfte geprägt werden, versuchen wir, mit unserem aktuell an der Johannes Kepler Universität angesiedelten Forschungsprojekt „Organisationale Resilienz in der COVID-19 Krise“, zu verstehen, wie Führungskräfte zur Resilienz ihrer Gesundheitsorganisation beitragen. Im Rahmen dieses Projekts führten wir Interviews mit Schlüsselpersonen in Deutschland, Israel, Italien, Österreich, Schweiz, Spanien und Taiwan, um die Situation im Gesundheitssystem besser zu verstehen und entsprechende Analysen vornehmen zu können. Zudem analysierten wir Zeitungsartikel zu COVID-19, mit dem Ziel die Situation aus einer breiteren (nationalen) Perspektive zu erfassen, und um Informationen über Faktoren zu sammeln, die die Situation im Gesundheitswesen potentiell beeinflussen, wie z.B. freigegebene Schutzmaßnahmen oder gesellschaftliche Reaktionen. über hinaus müssen Führungskräfte aber natürlich auch auf ihre eigene Resilienz achten, insbesondere da wir aus vergangenen Pandemien, wie dem SARS-Ausbruch im Jahr 
2003, wissen, dass es zu psychologischen (Langzeit-) Auswirkungen kommen kann ${ }^{15}$. Betrachtet man nun die Entwicklung der aktuellen COVID-19 Pandemie, so zeigen unsere ersten Studienergebnisse, dass das Mindset unserer befragten Führungskräfte zunächst stark von der Einstellung geprägt war, dass dieses neuartige Virus uns hier in Europa nicht signifikant betreffen würde und somit eher, wie bereits bei dem SARS Ausbruch in den Jahren 2002/2003, ein Problem des asiatischen Raums sei. Dieses Mindset wurde einerseits durch Erfahrungen mit früheren Virusausbrüchen genährt, andererseits aber auch durch die Politik und Presse. Diese zeigten sich Anfang 2020 ausgesprochen zuversichtlich und betonten, dass davon ausgegangen würde, dass Länder wie Deutschland Österreich und die Schweiz - wie es bei vorherigen Virusausbrüchen auch der Fall gewesen sei - von COVID-19 maximal durch Einzelfälle betroffen sein würden.

Hierzu berichten Der Standard am 28. Januar 2020 sowie die Neue Zürcher Zeitung am 24. Januar 2020:

\section{„In Europa droht laut Experten mit hoher Wahrscheinlichkeit »keine signifikante Gefähr- dung " "16, "Weltgesundheitsorganisation sieht Notlage auf China beschränkt"17}

Zudem wurde das Virus teilweise stark verharmlost, wodurch mögliche Vorbereitungsmaßnahmen als tendenziell weniger relevant erschienen. Diesbezüglich schrieb Die Zeit am 30. Januar:

\section{„Das Coronavirus 2019-nCoV ist - das zeigen erste Daten - klinisch vermutlich harmloser als seine berüchtigten Vorgänger Sars und Mers. "18, „Obwohl die täglich steigenden Fall- zahlen aus China und die stetig wachsende Zahl von betroffenen Ländern bedrohlich wir- ken, ist das Virus offenbar kein geborener Weltenbummler. "19}

Weiterhin betonten Politik und Presse die gute Vorbereitung und Überlegenheit ihrer nationalen Gesundheitssysteme. In diesem Sinne berichtete Die Zeit am 30. Januar 2020:

„Seit 2005 soll in einem solchen Krisenfall ein nationaler Pandemieplan helfen, der regelmäßig überarbeitet wird, zuletzt 2016. Schon vor Tagen hat das Robert Koch-Institut (RKI) Pläne veröffentlicht, was Ärzte und Gesundheitsämter im Falle eines CoronavirusFalles tun sollen. (...) »Die Zuständigkeiten sind sehr klar geregelt. "'20

Erst als die Infektionszahlen stiegen, die Situation im italienischen Bergamo anfing außer Kontrolle zu geraten und auch die Meinung in Politik und Presse sich wandelte, stellten unsere befragten Führungskräfte ihr vorherrschendes Mindset in Frage und fingen an, besonders auch auf politischen Druck hin, Vorbereitungen für eine nun vorstellbare Pandemie zu treffen. Etwas früher reagierten diejenigen, die berufliche Beziehungen nach China und/oder Italien pflegten und somit die Informationen aus erster Hand bekamen. Hierzu berichtete das Zeit Magazin über die Erfahrungen von drei IntensivärztInnen aus dem Frühjahr 2020: 


\section{„Die Stimmung im Krankenhaus ist leicht angespannt. Die Bilder aus Italien und die Be- richte von Kollegen dort beunruhigen uns." 21}

Obwohl die Gefahr einer möglichen Pandemie seit Jahren bekannt ist, konnten wir ein relativ einheitliches und außerordentlich hartnäckiges Meinungsbild bei unseren befragten Führungskräften feststellen. Und auch mit dem Ausmaß der zweiten Welle, welche ab Oktober über Deutschland, Österreich und die Schweiz rollte und vor welcher von verschiedenen Stellen gewarnt wurde, hätte laut der Mehrheit unserer befragten Führungskräfte niemand rechnen können. Während zwar im Unterschied zur ersten Welle im Frühjahr wesentliche Probleme wie bspw. der Mangel an Masken, Schutzausrüstung und Beatmungsgeräten gelöst wurden und unsere Befragten auch von Fortschritten in der Behandlung von COVID-19 berichteten, wurde der Sommer nur von wenigen genutzt, um die bisherige Krise auszuwerten und sich umfangreich auf

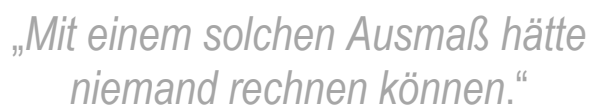
den bevorstehenden Winter vorzubereiten.

Hinsichtlich der akuten Bewältigungsphase, im April 2020 sowie ab Oktober 2020, berichtete der Großteil unserer befragten Führungskräfte von einer extremen Arbeitsbelastung, wodurch es ihnen kaum noch möglich war, auf ausgleichende Aktivitäten zurückzugreifen. Zudem waren natürlich auch die befragten Führungskräfte von den Einschränkungen des öffentlichen Lebens betroffen, wodurch sie auf ihre gewohnten Ausgleichsmöglichkeiten wie bspw. "mit Freunden essen gehen“ verzichten mussten. Bedingt durch die starke Arbeitsbelastung und dem Wegfall der sonst üblichen Ausgleichmöglichkeiten, griffen die meisten unserer befragten Führungskräfte auf bereits in der Vergangenheit aufgebaute Resilienzressourcen zurück, berichteten aber gleichzeitig - aufgrund der Dauer der Krise und fehlenden resilienzaufbauenden Aktivitäten von einem stetigen Verfall dieser Ressource.

Betrachtet man Krisen in Krankenhäusern in der Vergangenheit, so lässt sich die angewandte Bewältigungsstrategie auf die hier erprobten kurzfristigen und ggf. katastrophenartigen Krisen, wie bspw. Massenunfälle zurückführen. Obwohl diese Arten von Krisen den Betroffenen einiges abverlangen, sind diese in absehbarer Zeit zu bewältigen, worauf die Möglichkeit der Erholung folgt. Eine solche Strategie ist aber für die Bewältigung einer lang andauernden Krise, wie es die COVID-19 Krise darstellt, ungeeignet und birgt ein erhebliches Risiko für Erschöpfung, Burnout und Depression. Darüber hinaus können die negativen Emotionen, die bei ungünstiger oder fehlender Bewältigung, zu einer Abwärtsspirale führen, die Resilienz der Betroffenen, im Gegensatz zu positiven Emotionen, welche sich als förderlich für die Resilienz erweisen können ${ }^{22}$, zusätzlich hemmen. Ein weiteres Problem, welches wir im Rahmen unserer Interviews identifizieren konnten, resultiert aus dem Virus selbst. Auch wenn Komplikationen und Todesfälle einen Teil der Medizin darstellen, so sind es ÄrztInnen und PflegerInnen dennoch in der Regel gewohnt, Erfolg mit den angewandten Therapien zu haben. Bei 
COVID-19 stoßen sie allerdings an ihre Grenzen, da einerseits nach wie vor wenig über das Virus bekannt ist, anderseits klassische Behandlungsmethoden nicht anschlagen. Dieses führt zu einem Gefühl des Ausgeliefertseins und der Ohnmacht gegenüber der Krankheit, welches einem auch die eigene Verletzlichkeit bewusstwerden lässt.

Zusammenfassend werden in der nachfolgenden Infobox die Kernerkenntnisse aus unserer Studie anhand von vier identifizierten Handlungsfeldern dargestellt.

\section{Fazit}

Während unsere Gesundheitssysteme weiterhin auf die COVID-19 Pandemie reagieren, sollte Resilienz zur Kernphilosophie innerhalb der Organisationen werden, um sicher zu stellen, dass sie in der Lage sind, trotz Unterbrechungen wie COVID-19, zu funktionieren. Weg von einem Wettlauf um Ressourcen hin zu Systemen, die bereit sind, bei gleichzeitiger Aufrechterhaltung grundlegender Dienstleistungen, zu lernen und zu wachsen. Krisenvorsorge ist essentiell für Mitarbeitende, die selbst einem erhöhten Risiko ausgesetzt sind, bis hin zu ManagerInnen, GesetzgeberInnen sowie PolitikerInnen, die eine Verantwortung für die Gesundheit und Sicherheit ihrer MitarbeiterInnen und der breiten Öffentlichkeit tragen. Denn zusätzlich zu den möglichen physischen und psychologischen Folgen, können Pandemien in Krankenhäusern die Leistung der allgemeinen Gesundheitsversorgung beeinträchtigen, was zu einer Bedrohung der öffentlichen Gesundheit führen kann. Beachtet man die ausgeprägte Mobilität und Internationalität unserer Gesellschaft und Wirtschaft, so gilt es für die Zukunft vorbereitet zu sein, denn die nächste Pandemie könnte bereits auf dem Vormarsch sein und niemand weiß um welche Art von Virus es sich diesmal handelt.

\section{Förderungserklärung}

Diese Publikation entstand im Rahmen des von der JKU Business School geförderte Forschungsprojektes „Organisationale Resilienz in der COVID-19 Krise (COVRES)“. 


\section{! Was können wir schon jetzt für den Umgang mit zukünftigen Pandemien lernen?}

\section{(1) Antizipation und ein offenes Mindset}

Um langfristig resilient zu sein, müssen Führungskräfte Krisen jedweder Art antizipieren und die daraus möglicherweise entstehenden Konsequenzen abwägen können. Die aktuelle COVID-19 Krise hat gezeigt, dass die Antizipation und die daraus folgenden Vorbereitungen eher verzögert vorgenommen wurden. Um sich auf potenzielle, wenn auch zum jeweiligen Zeitpunkt als unwahrscheinlich eingestufte Gefahren vorzubereiten, bedarf es einer guten Mischung aus Erfahrung sowie einem offenen Mindset, sodass auf Unerwartetes bzw. Unbekanntes adäquat reagiert werden kann. Denn wie die COVID-19 Pandemie eindrucksvoll gezeigt hat, können auch Ereignisse mit einer tendenziell geringen Eintrittswahrscheinlichkeit schwerwiegende Folgen haben.

\section{(2) Strukturelle Probleme}

Die Studienergebnisse haben gezeigt, dass die COVID-19 Krise wie ein Brennglas auf die bereits langjährig bekannten strukturellen Probleme des Gesundheitssystems wirkt. Als Beispiele seien hier der chronische Personalmangel in der Pflege genannt sowie die fehlende Digitalisierung in nahezu jedem Bereich unseres Gesundheitssystems. Nur wenn solche Probleme nachhaltig gelöst werden, können wir zukünftige Pandemien besser bewältigen. Dies setzt einen gewissen Einsatz der EntscheidungsträgerInnen der Gesundheitspolitik voraus. Darüber hinaus zeigt die COVID-19 Krise ebenfalls klar auf, wo Probleme innerhalb einer Organisation bestehen, sei es in Form eines schlechten Arbeitsklimas oder im Rahmen fehlender Kooperation und Absprachen. Auch hier sind Führungskräfte gefragt in-house Lösungen zu finden, um zukünftig Krisen und stressige Situationen besser bewältigen zu können.

\section{(3) Vernetzung}

Gesundheitsorganisationen, die besonders gut vernetzt sind, waren besser auf die anrollende Pandemie vorbereitet. Durch Kontakte mit KollegInnen in China und Italien, verfügten diese Führungskräfte häufig über mehr Informationen z.B. bezüglich des richtigen Anlegens von Schutzkleidung und konnten diese Informationen an ihre Teams weitergeben, wodurch diese sich und ihre PatientInnen besser schützen bzw. versorgen konnten. Um zukünftige Pandemien besser bewältigen zu können, sollten Gesundheitsorganisationen folglich stärker darauf achten, sich zu vernetzten, international sowie national. Unsere befragten Führungskräfte wiesen teilweise sogar auf eine fehlende Vernetzung innerhalb eines Bundeslandes sowie innerhalb eines Krankenhauses hin. Gerade in Pandemiezeiten und im Umgang mit einer unbekannten Krankheit sollte versucht werden, aus den unmittelbar gemachten Erfahrungen der anderen zu lernen.

\section{(4) Individuelle Resilienzstrategien}

Eine lang andauernde Krise wie eine Pandemie erfordert andere Bewältigungsstrategien als ein kurzfristiger Katastrophenfall. Trotz hoher Arbeitsbelastung muss es den Betroffenen möglich sein, kurzfristig abzuschalten und die Akkus wieder aufzuladen. Einerseits müssen die Ressourcen gegeben sein, um dies zu bewerkstelligen, anderseits müssen aber auch die Betroffenen selbst Alternativen zu ihren sonst praktizierten Abschaltungsmethoden finden. Insgesamt hat sich gezeigt, dass diejenigen, die sich vor der Pandemie mit diesen Themen auseinandergesetzt haben, auch für diese lange Krise besser gewappnet waren. Weiterhin identifizierten wir die Bewältigung von Emotionen als ein wichtiges und wiederkehrendes Thema. Negative Emotionen können die Resilienz der Führungskraft substantiell gefährden, dennoch lassen sie sich in Zeiten wie diesen kaum verhindern. Ein geschulter Umgang kann hier Abhilfe schaffen. 


\section{Literatur}

1 WHO (2019). Ten threats to global health in 2019. https://www.who.int/news-room/feature-stories/tenthreats-to-global-health-in-2019, first accessed March 2020.

2 Rouleau, L., Hällgren, M., \& de Rond, M. (2020). Covid-19 and Our Understanding of Risk, Emergencies, and Crises. Journal of Management Studies, 1-4.

3 Duchek, S. (2020). Organizational resilience: a capability-based conceptualization. Business Research, 13(1): 215-246.

4 Linnenluecke, M. K., \& Griffiths, A. (2012). Assessing organizational resilience to climate and weather extremes: complexities and methodological pathways. Climatic change, 113(3): 933-947.

5 Hartmann, S., Weiss, M., Newman, A., \& Hoegl, M. (2020). Resilience in the workplace: A multilevel review and synthesis. Applied Psychology, 69(3): 913-959.

6 Lengnick-Hall, C. A., Beck, T. E., \& Lengnick-Hall, M. L. (2011). Developing a capacity for organizational resilience through strategic human resource management. Human Resource Management Review, 21(3): 243255.

7 Pearson, C. M., \& Clair, J. A. (1998). Reframing crisis management. Academy of management review, 23(1): 59-76.

8 Avey, J. B., Avolio, B. J., \& Luthans, F. (2011). Experimentally analyzing the impact of leader positivity on follower positivity and performance. The Leadership Quarterly, 22(2): 282-294. Gooty, J., Gavin, M., Johnson, P. D., Frazier, M. L., \& Snow, D. B. (2009). In the eyes of the beholder: Transformational leadership, positive psychological capital, and performance. Journal of Leadership \& Organizational Studies, 15(4): 353-367. Walumbwa, F. O., Peterson, S. J., Avolio, B. J., \& Hartnell, C. A. (2010). An investigation of the relationships among leader and follower psychological capital, service climate, and job performance. Personnel Psychology, 63(4): 937-963.

9 Sutcliffe, K. M., Paine, L., \& Pronovost, P. J. (2017). Re-examining high reliability: actively organizing for safety. BMJ quality \& safety, 26(3): 248-251. Williams, T. A., Gruber, D. A., Sutcliffe, K. M., Shepherd, D. A., \& Zhao, E. Y. (2017). Organizational response to adversity: Fusing crisis management and resilience research streams. Academy of Management Annals, 11(2): 733-769.

10 Iseringhausen O., Staender J. (2012). Das Krankenhaus als Organisation. In: Apelt M., Tacke V. (eds) Handbuch Organisationstypen. VS Verlag für Sozialwissenschaften.

11 Lengnick-Hall, C. A., Beck, T. E., \& Lengnick-Hall, M. L. (2011). Developing a capacity for organizational resilience through strategic human resource management. Human Resource Management Review, 21(3): 243255.

12 Avey, J. B., Avolio, B. J., \& Luthans, F. (2011). Experimentally analyzing the impact of leader positivity on follower positivity and performance. The Leadership Quarterly, 22(2): 282-294. Gooty, J., Gavin, M., Johnson, P. D., Frazier, M. L., \& Snow, D. B. (2009). In the eyes of the beholder: Transformational leadership, positive psychological capital, and performance. Journal of Leadership \& Organizational Studies, 15(4): 353 ?367. Walumbwa, F. O., Peterson, S. J., Avolio, B. J., \& Hartnell, C. A. (2010). An investigation of the relationships among leader and follower psychological capital, service climate, and job performance. Personnel Psychology, 63(4): 937-963.

13 Williams, T. A., Gruber, D. A., Sutcliffe, K. M., Shepherd, D. A., \& Zhao, E. Y. (2017). Organizational response to ad-versity: Fusing crisis management and resilience research streams. Academy of Management Annals, 11(2): 733-769.

14 Soucek, R., Pauls, N., Schlett, C. (2018). Resiliente Führung. Zeitschrift für Führung und Organisation, 01/2018: 9-13.

15 Maunder, R. G., Lancee, W. J., Balderson, K. E., Bennett, J. P., Borgundvaag, B., Evans, S., et al., (2006). Longterm psychological and occupational effects of providing hospital healthcare during SARS outbreak. Emerging infectious diseases, 12(12), 1924-1932. Tam, C. W., Pang, E. P., Lam, L. C., \& Chiu, H. F. (2004). Severe acute respiratory syndrome (SARS) in Hong Kong in 2003: stress and psychological impact among frontline healthcare workers. Psychological medicine, 34(7): 1197-1204. Perrin, P. C., McCabe, O. L., Everly, G. S., \& Links, J. M. (2009). Preparing for an influenza pandemic: mental health considerations. Prehospital and disaster medicine, 24(3): 223-230.

16 Der Standard, (2020). Neue Viren-Verdachtsfälle gemeldet. Januar 28(2354). 


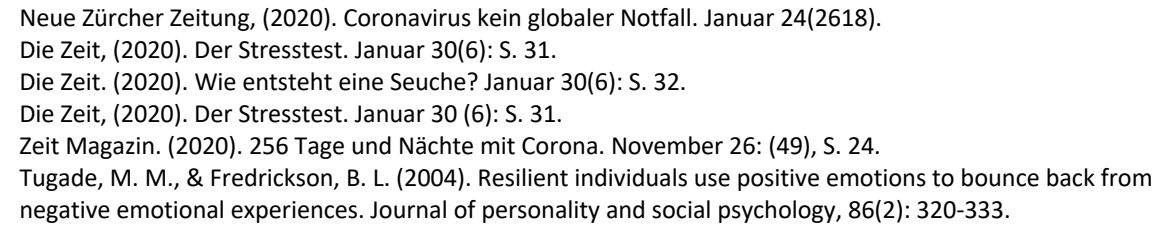

\section{Information zu den Autorinnen}

Jun.-Prof. ${ }^{\text {in }}$ Dr. ${ }^{\text {in }}$ Charlotte Förster ist Juniorprofessorin für Europäisches Management an der Technischen Universität Chemnitz. Aufbauend auf ihren Forschungsaktivitäten an der Johannes Kepler Universität Linz beschäftigt sich sie sich aktuell mit Pandemiemanagement. Ihre Forschungsschwerpunkte liegen im Bereich Führung, Resilienz von Führungskräften sowie organisationale Resilienz mit besonderem Fokus auf Gesundheitsorganisationen. Für nähere Informationen besuchen Sie: www.charlotte-foerster.com.

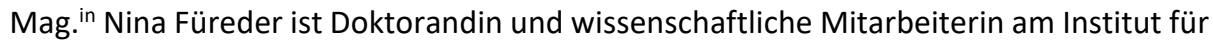
Leadership und Change Management an der Johannes Kepler Universität (JKU) Linz. Ihr besonderes Interesse und ihre Forschungsschwerpunkte liegen bei der Weiterentwicklung der Patientensicherheit und Sicherheitskultur im Krankenhaus, der Implementierung von innovativen technischen Lösungen sowie Resilienzmanagement im Gesundheitswesen. 